\title{
Coulisses
}

Revue de théâtre

25| Hiver 2002

Varia

\section{Du « Centre d'Art et de Plaisanterie » à « L'Allan »}

Interview du nouveau directeur de la scène nationale de Montbéliard, Didier Levallet par Raphaëlla Rosello, Julienne Turan et Ruth Trommenschlager, étudiants, DEUG L. M.

\section{(2) OpenEdition}

12 Journals

Édition électronique

URL : http://journals.openedition.org/coulisses/5899

DOI : $10.4000 /$ coulisses.5899

ISSN : 2546-9460

Éditeur

Presses universitaires de Franche-Comté

\section{Édition imprimée}

Date de publication : 1 janvier 2002

Pagination : 9-13

ISBN : 2-84627-052-X

ISSN : $1150-594 X$

\section{Référence électronique}

« Du « Centre d'Art et de Plaisanterie » à «L'Allan » », Coulisses [En ligne], 25 | Hiver 2002, mis en ligne le 24 octobre 2019, consulté le 15 novembre 2019. URL : http://journals.openedition.org/coulisses/5899 ; DOI : 10.4000/coulisses.5899

Ce document a été généré automatiquement le 15 novembre 2019.

Coulisses 


\section{$\mathrm{Du}$ « Centre d'Art et de Plaisanterie ${ }^{1}$ » à « L'Allan »}

Interview du nouveau directeur de la scène nationale de Montbéliard, Didier Levallet par Raphaëlla Rosello, Julienne Turan et Ruth Trommenschlager, étudiants, DEUG L. M.

Ayant entrepris des études de journalisme, ancien professeur de Conservatoire de musique, organisateur du festival de jazz annuel de Cluny et musicien de grande notoriété, il est désormais le directeur de L'ALLAN, scène nationale de Montbéliard. Nous remercions Didier LEVALLET pour son accueil plus que chaleureux et du temps qu'il nous a consacré malgré son planning très chargé.

\section{COULISSES - COMMENT S'EST dÉROULÉ CE QUE VOUS APPELEZ LE RENOUVEAU de LA SCÈNE NATIONALE DE MONTBÉLIARD?}

D. L. - La scène nationale est un lieu pluridisciplinaire qui doit présenter des spectacles de toute nature. Au début, il s'agissait d'action culturelle qui conduisait à une sensibilisation du public et à un apport pratique avec des ateliers. André MALRAUX, premier ministre de la Culture, a inventé le principe des Maisons de la Culture en parallèle de la décentralisation des scènes qui sont désormais installées dans des villes telles que Le Havre, Amiens ou encore Bourges... L'objectif étant de rendre accessible la culture à tous. Duhamel a ensuite créé des Centres d'action culturelle comme à Montbéliard. Cette décentralisation était tout d'abord destinée au théâtre mais les habitudes culturelles se sont modifiées et la culture s'est donc diversifiée. De la musique, pourquoi pas? surtout qu'en tant que musicien, Didier Levallet a souffert du manque d'ouverture à la musique.

\section{C. - POURquol AVOIR CHOISI LA scène de MONTBÉlIARd? EST-Ce PAR MOtIVATION} PERSONNELLE?

D. L. - Il y a deux raisons: Montbéliard est un axe fort concernant la musique, il s'agissait ensuite de choisir un directeur en fonction d'un projet qu'il proposait afin de réorienter la scène nationale. Ce nouveau directeur a choisi la ville de Montbéliard car il connaissait déjà la région pour y avoir fait diverses résidences ainsi que des concerts. Ayant carte blanche pour réorienter la scène en l'ouvrant sur la musique et disposant de structures, d'outils et d'un théâtre relativement bien équipé qui se 
complète avec Le Palot, ancien cinéma à l'esprit magique des années 20, il a accepté le challenge, cette aventure singulière.

\section{C. - quelle sera la place accordée à la musique dans vos choix de PROgRAMMATION?}

D. L. - Parmi les structures dont dispose Didier Levallet, il y a deux salles : un théâtre du XIX ${ }^{e}$ siècle, à l'italienne ainsi qu'une salle Le Palot. Ce cabaret est un lieu ouvert aux musiques actuelles (rock, jazz, reggae).

La musique participe à un renouveau de la scène de Montbéliard après le départ de la Cie du Théâtre de rue qui la dirigeait et dont la dernière plaquette de programmation était une forme de faire-part signant la mort de la scène nationale. Cette ouverture à la musique proposée par le ministère est vécue comme une renaissance. On voit que pour la saison 2001-2002 une grande importance est accordée au jazz avec le collectif ZHIVARO (N.D.L.R. Didier Levallet en fait partie), le Charméditerranéen, Éric Le Lann, Steve Lacy, Louis Slavis... Importance aussi accordée aux musiques du monde avec le Quatuor Takacs, ...aux musiques, aux sons inédits, surprenants, et aussi le théâtre d'images sonores.

\section{C. - AVEZ-vous RENCONTRÉ des difficultés Afin d'ACCÉder À LA DIRECTION ? ICI IL S'AgIt de DIRIgER UN THÉÂtre AVEC TOUTES SORTES DE CONTRAINTES, DE RÈgLes ÉTABLIES...}

D. L. La difficulté majeure réside dans les problèmes de financement. Actuellement les subventions se partagent entre la ville et l'État, cas de figure assez exceptionnel puisqu'il devrait y avoir un troisième acteur tel que le département ou la région. Ces difficultés conduisent parfois à des choix douloureux puisque l'hôtel Sponek - lieu d'exposition - va fermer ses portes, une partie du personnel sera licenciée et le programme est moindre par rapport à l'ambition initiale.

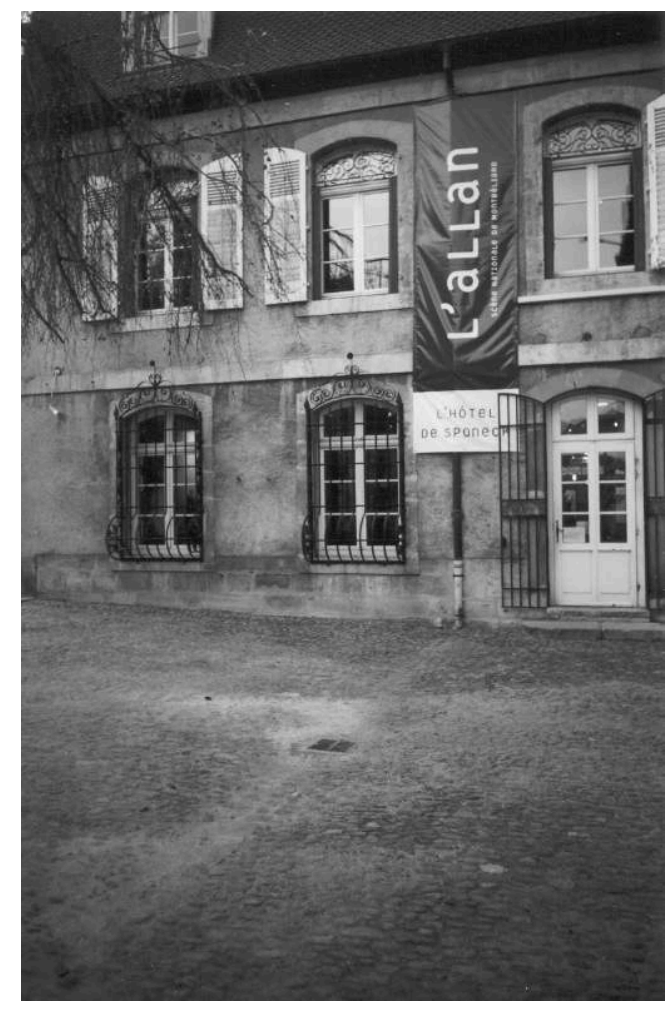

Photo R. Rosello 
C. - afin d'assurer la programmation de lallan, par Quelle Équipe Êtes-vous SECONDÉ ? POUVEZ-VOUS NOUS DIRE QUELQUES MOTS DE VOS PARTENAIRES ?

D. L. - Didier Levallet est entouré par la même équipe qui secondait ses prédécesseurs cependant un nouvel administrateur a pris place à ses côtés pour verrouiller l'organisation de la maison. "Le fou furieux ", " la tête chercheuse ", "l'éclaireur ", termes à connotations étranges voire mystérieuses, sont en fait les personnes qui se rendent à tous les spectacles afin d'être convaincus de la qualité des représentations afin de persuader à leur tour le public. Ce travail minutieux rend compte de la qualité de la scène nationale de Montbéliard.

C. - AU SUJet des partenaires, Quels liens souhaitez-Vous tisser ou Renouveler

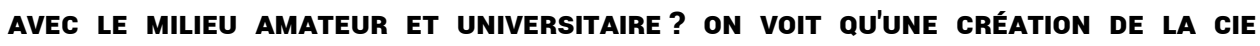
CARCANA SE faIt AVEC LA COLLABORATION d'UN LYCÉE TECHNIQUe ET LES ÉlÈVES SONT par ailleurs conviés auX ateliers de chant, musique, danse, Jonglage... est-Ce VOTRE POLITIQUE ? TISSER DES LIENS AUTOUR D'UN ÉVÉNEMENT CULTUREL ?

D. L. - Oui, il faut susciter et répondre à leur demande. Comme par exemple la classe du lycée des Huisselets qui pour son journal se rend à diverses représentations pour en faire une critique, le but étant de favoriser l'accès aux activités artistiques pour un public divers. Il s'agit de sensibiliser le public afin de changer ses habitudes, de lui donner goût à l'art. C'était la mission de Malraux et c'est ce que Didier Levallet s'efforce de faire.

C. - VOUS AVEZ DIT QUE LE PLUS BEAU COMPLIMENT QUE L'ON VOUS A FAIT DANS VOTRE VIE DE MUSICIEN EST LE SUIVANT : « NOUS VOULONS VIVRE, COMME CET ORCHESTRE JOUE ».

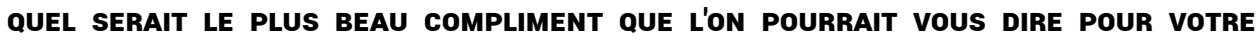
ENgAgEMENT DANS LE THÉÂTRE ?

D. L. - «Je me suis reconnu » voilà le plus beau compliment que l'on pourrait lui adresser. C'est le principe de Roman de famille pièce créée par le public, pour le public et grâce au public.

C. - pouvez-vous nous dire quelques mots AU SUJet des RÉsidences ? IL Y A BEAUCOUP DE RÉSIDENCES MUSICALES OU THÉÂTRALES.

D. L. - Le principe des résidences est d'organiser la présence d'artistes qui travaillent sur place afin de sensibiliser un plus large public, le faire venir, accrocher son intérêt, lui tendre la main. Elles permettent d'assurer une présence artistique continue afin de tisser des liens de proximité, car le théâtre ne s'adresse plus à une élite ; il faut dénouer cette relation un peu crispée, l'emmener plus loin que son attente pour le surprendre sans le froisser. Il y a une initiation à tout, il s'agit d'un don de soi afin de susciter son intérêt.

C. - ON peut penser que vous êtes parvenu à différencier la scène de MONTBÉlIARD PUISQUE :

- primo, vous êtes musicien

- secundo, la plaquette de présentation est plus qu'originale, elle est poétique

- tertio, le nom de la scène, I'Allan, porte sens

Ce particularisme, le revendiquez-vous ? Est-ce une manière de vous affırmer, vous et votre scène?

«Les Biefs » était le premier nom auquel Didier Levallet avait pensé pour baptiser la scène nationale puisque les biefs sont des cours d'eau qui relient deux lieux et qu'il voulait montrer le lien entre les différents lieux culturels de Montbéliard. De plus, il était nécessaire de marquer une rupture complète avec ce qu'il y avait avant; la 
nouvelle plaquette d'« un style classe » et les banderoles rouges sur le bâtiment de l'hôtel Sponek marque la renaissance du théâtre de Montbéliard.

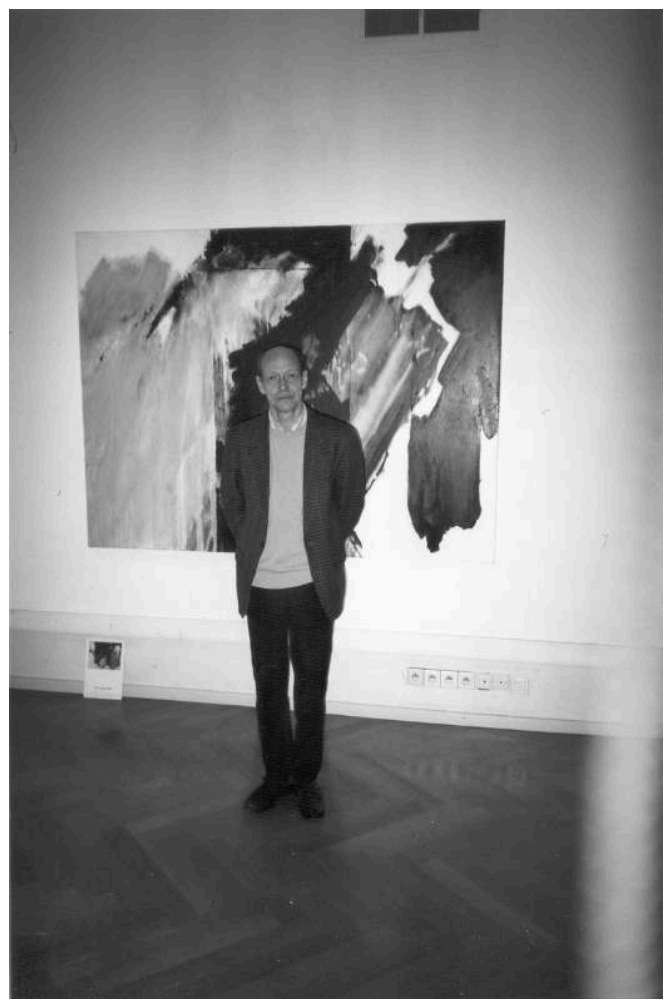

Photo R. Rosello

C. - NOUS AVONS tenté d'INTERPRÉter VOtRe PLAQUetTe SUR Le thème de L'EAU de LA MANIÈRE SUIVANTE : VOUS SEMBLEZ ACCORDER UNE PLACE IMPORTANTE AUX HOMMES, ACteurs et protagonistes de l'ARt, afin Qu'Ils tissent des liens Permettant L'ACCÈs DE LA CULTURE À TOUS. L'EAU, LA RIVIÈRE SONT DES SYMBOLES DE LA CULTURE SOUS SES DIFFÉRENTS ASPECTS, CALME, VIOLENTE À L'IMAgE DU THÉÂTRE TANTÔT DANS LA NORME, et tantôt Plus engagé, plus grinçant. LA scène de L'allan Établirait des PONTS, des passerelles entre les hOMmes, QUI ONT des Centres d'intérêts DIFFÉRENTS, de LA MUSIQUe À LA DANSE, EN PASSANT PAR LE THÉÂTRE. EST-CE LÀ L'OBJECTIF ? L'AMBITION DE VOTRE SCÈNE ?

D. L. - Le baptême de la scène nationale s'est déroulé le 14 septembre dernier, - jour du deuil européen-, il s'agissait de savoir s'il fallait ou non annuler cette inauguration, afin de ne pas blesser la population. Elle a été maintenue et lors de son discours, Didier Levallet s'est expliqué : «Si l'art, la culture étaient de la pure distraction, alors il aurait fallu annuler cette manifestation mais l'art est signe de civilisation, donc, contraire à la barbarie, alors notre présence était nécessaire ».

C. - LA RELATION ENTRE THÉÂTRE ET MUSIQUE EST VISIBLE DANS VOTRE PROgRAMMATION. UNE AUTRE CORRESPONDANCE, EST PERCEPTIBLE, CELLE DE LA DANSE. LES MOTS PEUVENTILS SE DESSINER AVEC LE CORPS, L'EXPRESSION SCÉNIQUE DEVENANT UN LANgAgE PRESQUE UNIVERSEL?

D. L. - La civilisation française est sous la dictature du cartésianisme, du livre écrit qui est sacralisé et donc du langage conceptuel. Dans la danse et la musique, il y a aussi un langage écrit (partition, notes). Mais il y a une plus grande liberté de sens d'interprétation. Les émotions, le mouvement du corps, l'espace occupé par les 
danseurs sont magiques, plus purs. La danse est le rapport entre l'art abstrait et l'art du sublimatoire.

\section{C. - Un changement de carrière est-IL prévu prochainement ? Écriture ? PEINTURE ? RÉALISATION?}

Didier Levallet désire tout d'abord mener sa carrière de musicien en parallèle avec celle de directeur de théâtre. Aimant l'imprévu, la vie, donc un peu nomade, il dispose, s'il le désire, d'un contrat de neuf ans à la tête de l'Allan; sachant qu'il lui faut trois années afin d'instaurer son projet, puis trois autres, afin de le faire vivre.

D'autre part, il s'est donné comme pari de donner un outil tel que l'Allan à la musique, de créer un précédent.

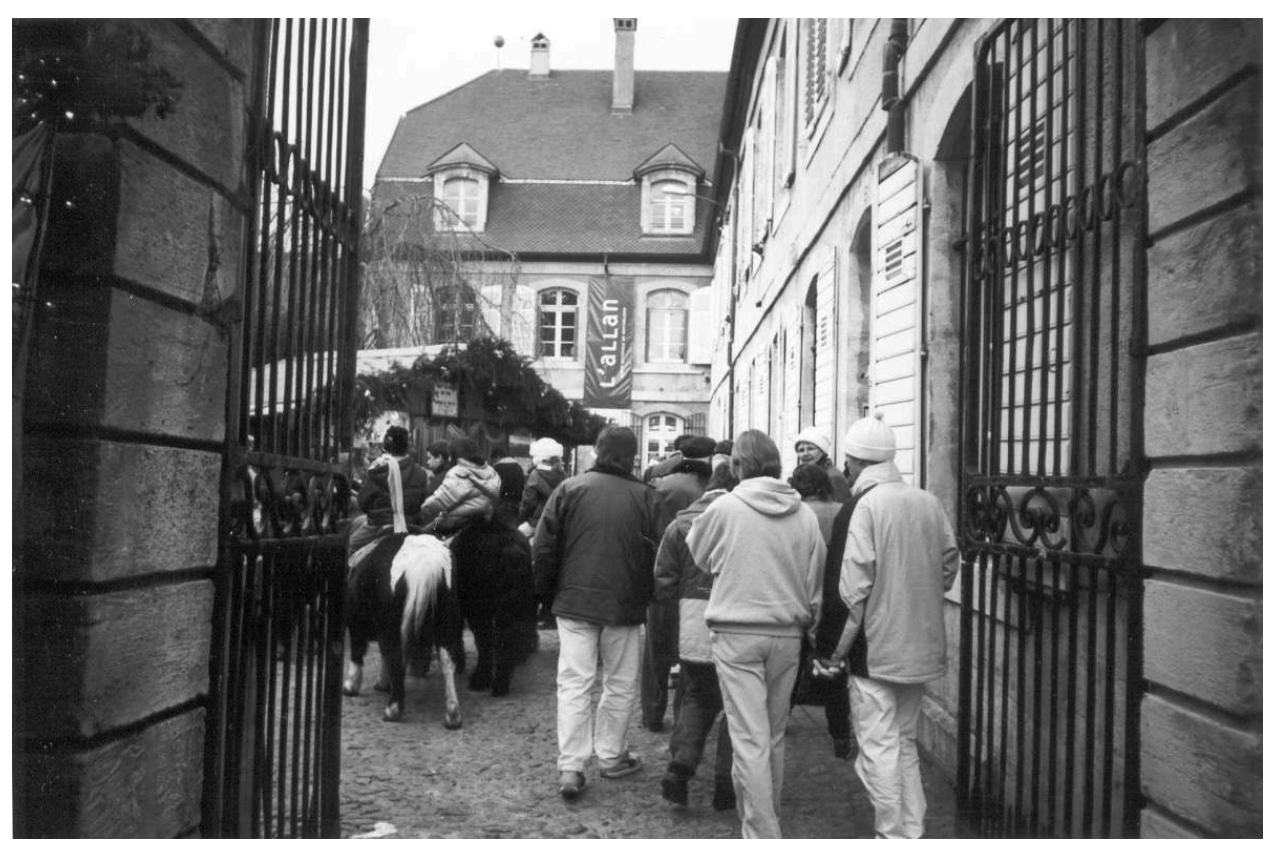

Photo R. Rosello

\section{NOTES}

1. Coulisses a régulièrement rendu compte des activités du Centre d'Art et de Plaisanterie. 